\title{
Autologous Costal Cartilage Graft-A New Method to Treat Articular Cartilage Defects: Case Report and Note of Surgical Technique
}

\author{
Antonio Gigante ${ }^{1}$ Marco Cianforlini ${ }^{1} \quad$ Luca Farinelli $^{1} \quad$ Riccardo Girotto $^{2} \quad$ Alberto Aquili $^{1}$ \\ ${ }^{1}$ Clinical Orthopaedics, Department of Clinical and Molecular \\ Science, Università Politecnica delle Marche, Ancona, Italy \\ 2 Maxillofacial Surgery Unit, Azienda Ospedaliero-Universitaria \\ Ospedali Riuniti, Ancona, Italy \\ Address for correspondence Antonio Gigante, MD, Clinical \\ Orthopaedics, Department of Clinical and Molecular Sciences, \\ Università Politecnica delle Marche., Via Tronto 10/A, 60126 Ancona, \\ Italy (e-mail: a.gigante@univpm.it).
}

Joints 2018;6:246-250.

\begin{abstract}
Keywords

- cartilage repair

- patella

- costal graft

- rib perichondrium

Full-thickness articular cartilage defects do not heal spontaneously. Several techniques have been developed to address this issue, but none resulted in the restitutio ad integrum of the articular cartilage. The most frequent sites of chondral lesion in the knee are medial femoral condyle and patella. The patellofemoral lesions are characterized by outcomes that are generally worse than those of tibiofemoral ones. To date, it has been well recognized the chondrogenic potential of rib perichondrium, and costal cartilage grafts have been extensively used in reconstructive surgery. Considering the need to find a gold standard technique to restore articular defect, we developed and here described a new technique to repair cartilage lesions of the knee using autologous costal cartilage graft with its perichondrium. This innovative surgical approach can be used to treat full thickness articular defects using autologous hyaline cartilage, making it possible to cover wide defects. This one step technique is low invasive, not technically demanding with minimal donor site morbidity and it has low costs. The long-term clinical efficacy of the method remains to be evaluated.
\end{abstract}

\section{Introduction}

Full-thickness articular cartilage defects do not heal spontaneously. ${ }^{1}$ Patients with isolated cartilage defects may not have clinically and functionally significant problems. However, most of them have early degenerative changes, pain, and disability. ${ }^{2}$ In the last years, different tissues and techniques have been developed to restore articular defects of the knee.

Microfractures, ${ }^{3}$ autologous chondrocyte implantation $(\mathrm{ACI}),{ }^{4}$ matrix-induced autologous chondrocyte implantation, ${ }^{5,6}$ autologous matrix-induced chondrogenesis (AMIC), ${ }^{7}$ AMIC improved with autologous bone marrow concentrate, ${ }^{8}$ mosaicplasty, ${ }^{9}$ osteochondral allograft transplantation ${ }^{10}$ and other techniques present limitations and no consensus regarding which method is superior has been obtained. ${ }^{11}$ In addition, irrespective of the surgical technique used, hyaline cartilage has been obtained only in selected cases, without restoring articular cartilage microarchitecture. ${ }^{5,12,13}$

After the medial femoral condyle, the patella is the second most common location in the knee for the occurrence of chondral lesions. ${ }^{14}$ Furthermore, despite the wide selection of technique available, it has been well established that in the patellofemoral compartment outcomes are generally worse than in the tibiofemoral joint. ${ }^{15}$ For these reasons, the best technique remains to be addressed; the main goal in managing patellar injuries should be to restore the cartilage surface with grafts of hyaline cartilage to re-establish the biomechanical properties of the tissue restoring knee function. The use of autologous costal cartilage graft for the treatment of articular defects of the knee in humans has never been received

March 18, 2018

accepted

August 6, 2019

published online

October 11, 2019
DOI https://doi.org/

$10.1055 / \mathrm{s}-0039-1697609$.

ISSN 2282-4324.
Copyright $\odot 2018$ Georg Thieme Verlag License terms

KG Stuttgart · New York

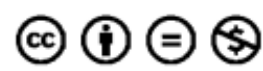


described before. Only limited and promising results have been obtained by animal models with costal fragments. ${ }^{16}$ Indeed, Du et al sliced rib cartilage into 3- to 5-mm fragments and then harvested cartilage grafts in the rabbit knee lesions in one step technique.

Considering this imperative, we developed a new technique for repair articular cartilage defect using autologous costal cartilage graft with its perichondrium.

The main elements contributing to the rationale for this choice were: (1) the graft is autologous and of hyaline nature; (2) the perichondrium of the graft has chondrogenic potential ${ }^{17}$; (3) the costal graft is widely used in reconstructive surgery $^{18,19}$ and usually it has a low donor site morbidity ${ }^{20}$; (4) the graft may be properly sized to cover large and fullthickness articular defects; (5) the method is "one-step" and has a low cost.

\section{Case Description}

In September 2017, a 15-year-old male was referred to the Department of Orthopaedics of the Polytechnic University of Marche (Italy) for anterior knee pain. Symptoms were exacerbated by going downstairs, running, jumping, and squatting. In addition, the patient described the movie sign, in which anterior pain was increased after prolonged sitting. No signs of patella instability were revealed. The Lachman test, anterior drawer test provided negative results. No signs of meniscal injuries were found. Magnetic resonance imaging demonstrated a focal cartilage defect in patella that seemed to be grade III according to the International Cartilage Repair Society (ICRS). ${ }^{21}$ Static and dynamic CT examination did not show relevant features of patellofemoral malalignment.

Patient had no history of malignancy, systemic inflammatory diseases, and neurologic or cardiovascular disorders. In addition, he agreed with a strict postoperative rehabilitation protocol.

Prior to surgery, patients failed the nonsurgical management of injections, bracing, and physical therapy that focused on quadriceps strengthening, peripatellar mobilization, core strengthening, abductor strengthening, and physiotaping.

After discussing with the patient and his parents about the possibilities of this new technique in comparison to other techniques, and after the signature of the informed consent, we elected to repair the diseased joint with an autologous costal cartilage graft.

\section{Surgical Treatment}

Under general anesthesia, with a tourniquet located in the thigh, a knee arthroscopy was performed. Under arthroscopy vision, we confirmed the diagnosis of patellar cartilage defect and excluded other possible lesions. We observed no meniscal injuries, cruciate ligaments ruptures, or osteoarthritis of both patellofemoral and tibiofemoral joint. The defect was classified grade III according to ICRS classification.

The unique anatomy of the patellofemoral joint makes surgical exposure to the patellar articular surface difficult to achieve arthroscopically; thus after the arthroscopic diagnosis, we preferred an arthrotomy through a lateral parapatellar incision that allowed a direct and perpendicular approach to the patellar lesion., ${ }^{6,22}$ With the patella everted, clear visualization of the defect was possible (-Fig. 1).

The surgical procedure commenced with the assessment and debridement of the full-thickness articular cartilage lesion. To debride the lesion, a curette and a motorized shaver were used to remove any unstable cartilage overlying or surrounding the chondral defect. The calcified cartilage layer at the base of the defect was removed using a curette to expose the subchondral bone.

An awl was used to make multiple small perforations in the exposed subchondral bone. The holes were performed $6 \mathrm{~mm}$ deep and placed 3 to $4 \mathrm{~mm}$ apart to ensure that the holes did not become confluent during the procedure. We began at the periphery and then progressed toward the center of the defect. The lesion measured $1 \times 1.5 \mathrm{~cm}$ (-Fig. 2).

Once measured the patellar defect by the ruler, we moved to the thorax to harvest the costal cartilage graft. A skin incision of approximately $3 \mathrm{~cm}$ was made along the major axis of the seventh rib in proximity of the right costosternal joint (-Fig. 3).

Separation of the overlying muscles was performed to expose the rib, a subperichondrial dissection of the seventh costal cartilage with delivery of the distal part of the rib was done and the required length was harvested. A 15-blade
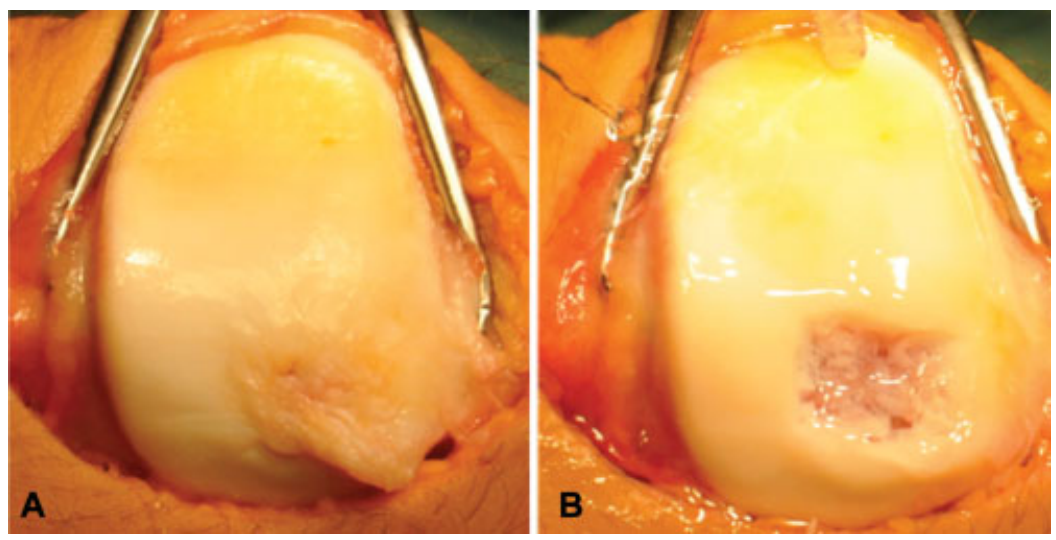

Fig. 1 (A) Cartilage defect of the medial facet of the patella. (B) The lesion after debridement. 

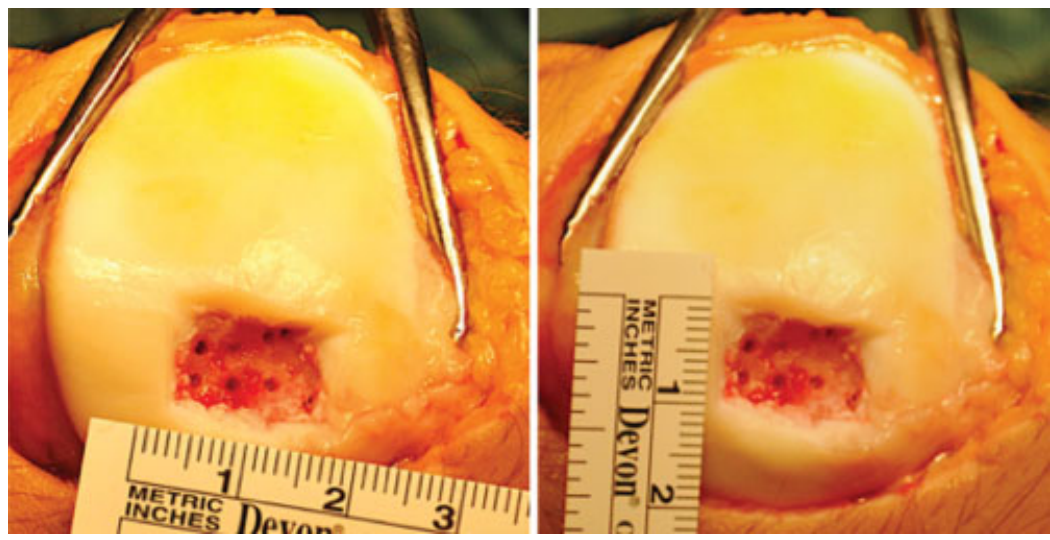

Fig. 2 Size of the chondral defect after microfractures.

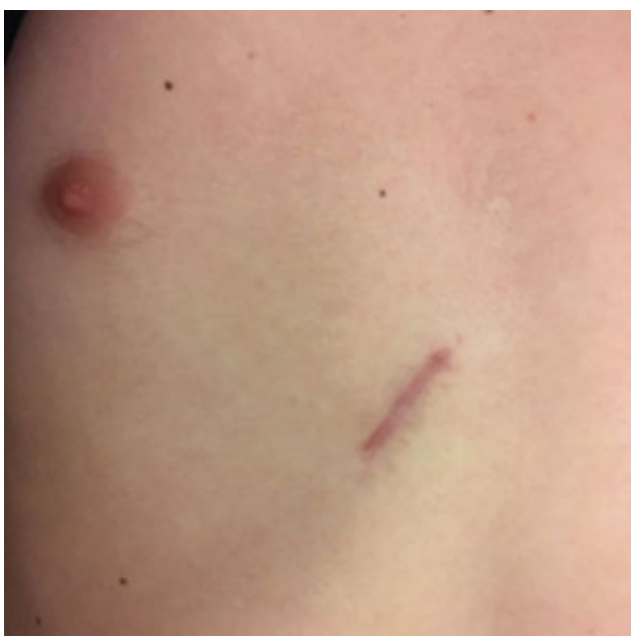

Fig. 3 Cutaneous scar at 3 months after surgery. The skin incision about of $3 \mathrm{~cm}$ was made along the major axis of the seventh rib in proximity of the right costosternal joint.

scalpel was then used to make incisions both at the medial and at the lateral aspect of the sized graft. An elevator was then used to remove the graft. The incisions should extend through the cartilage but not violate the underlying perichondrium. Indeed, special attention should be laid to preserve as intact the three sides of the rib (-Fig. 4).
The graft was carefully shaped and impacted into the defect. Its height matched with that of the neighboring cartilage, because an uneven articular surface could alter the biomechanical functionality of the patellofemoral joint. ${ }^{15}$ The graft was positioned with the perichondrium facing toward the trochlear groove (-Fig. 5).

To fix the graft in compression, we applied two nonabsorbable sutures through the graft; then we performed four holes across the patella. Subsequently, we put the free ends of the sutures through the patella and we tied two knots in the surface opposite to the patellofemoral joint. To ensure the graft in the lesion, we passed two absorbable sutures between perichondrium and the neighboring cartilage. After the implantation of the graft, the tourniquet was removed.

Lastly, we filled the borders of the implant with fibrin glue to make the surface smooth and to strengthen the graft. A drainage has been introduced to avoid local hematoma and then it was removed in the second postoperative day. The incisions were closed with absorbable sutures on the fascia and subcutaneous planes and with nonabsorbable sutures for the skin.

\section{Postoperative Care}

When he was discharged from the operating theater, the affected leg was immobilized in a brace in full extension for 48 hours; then continuous passive motion was used for the
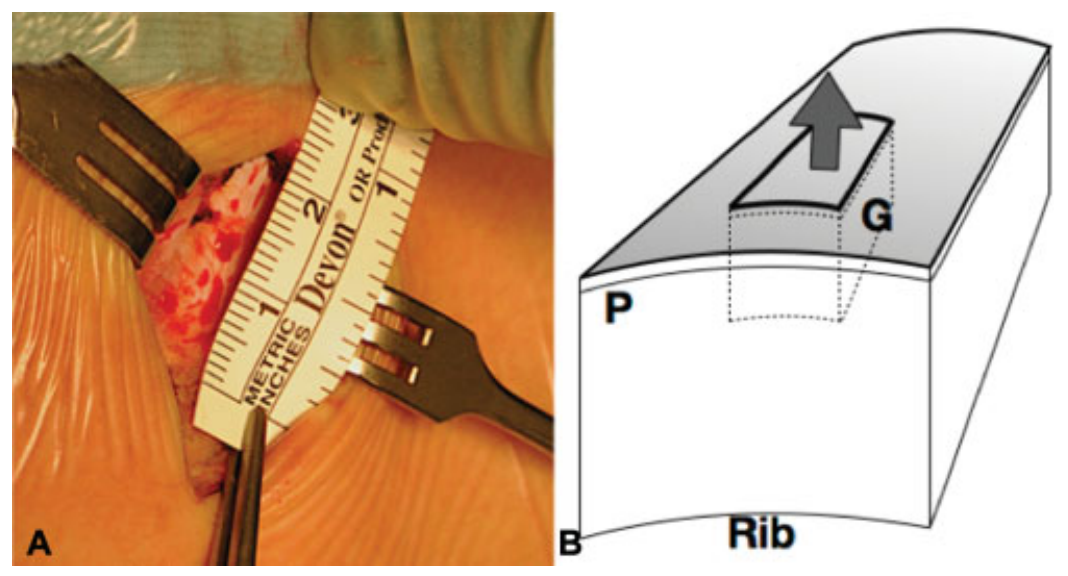

Fig. 4 (A) The surgeon measured the size of the graft. (B) Schematic representation of costal graft (G, Graft; P, perichondrium). 


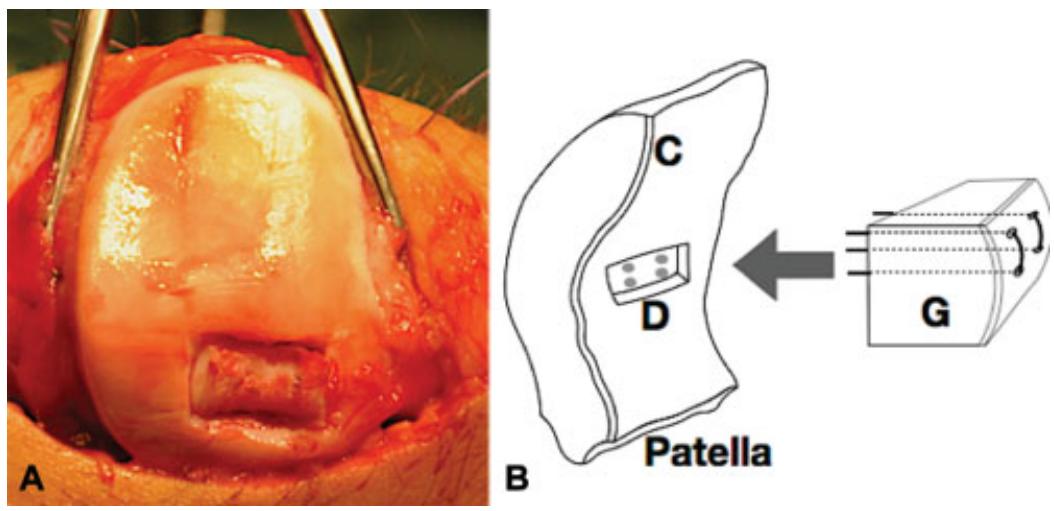

Fig. 5 (A) The implantation of the graft during surgery. (B) A schematic representation of implantation of the graft throughout nonabsorbable sutures across the patella (C, cartilage; $D$, defect; $G$, graft).

first month after surgery. Weight bearing was not allowed for 4 weeks, the patient was allowed to walk with crutches without putting weight on the affected leg. Progressive weight bearing on the affected limb and active movement during physiotherapy were allowed from the second month following surgery.

\section{Discussion}

We developed this technique on the basis of previous histological, immunohistochemical, and ultrastructure characterization of costal cartilage.

First, from a biological point of view, the costal cartilage represents the main source of hyaline cartilage in mammals and it is characterized by histological similarities to the articular cartilage. ${ }^{23}$ Furthermore, it is surrounded by rib perichondrium, whose chondrogenic capacity was demonstrated by Skoog et al in $1972 .{ }^{24}$ Summarizing, the graft presents the histochemical (hyaline) and microarchitectural features close to the articular cartilage; thus, it could support the biomechanical loading imposed by the joint with the peripheral perichondrium that might promote the integration mechanism. Moreover, the use of an autologous tissue avoids any adverse reactions.

Second, from a surgical point of view, it is easy to fit the costal graft in the lesion; in case of extensive or multiple defects, multiple costal cartilage grafts might be harvested from the same incision; it is a nondemanding and low invasive technique with minimal donor site morbidity. Lastly, the method proposed may be efficient in cost-benefit terms, because it is a "one step" and low cost procedure.

On the other hand, the method presents some critical issues. We do not know if the borders of the costal graft may be integrated in the neighboring cartilage and if the graft may be efficiently anchored to the bone through endochondral ossification. Moreover, the long-term clinical efficacy of the method remains to be evaluated.

Conflict of Interest

None declared.

\section{References}

1 Detterline AJ, Goldberg S, Bach BR Jr, Cole BJ. Treatment options for articular cartilage defects of the knee. Orthop Nurs 2005;24 (05):361-366

2 Richter DL, Schenck RC Jr, Wascher DC, Treme G. Knee articular cartilage repair and restoration techniques: a review of the literature. Sports Health 2016;8(02):153-160

3 Steadman JR, Rodkey WG, Briggs KK, Rodrigo JJ. [The microfracture technic in the management of complete cartilage defects in the knee joint]. Orthopade 1999;28(01):26-32

4 Brittberg M, Lindahl A, Nilsson A, Ohlsson C, Isaksson O, Peterson L. Treatment of deep cartilage defects in the knee with autologous chondrocyte transplantation. N Engl J Med 1994;331(14): 889-895

5 Enea D, Cecconi S, Busilacchi A, Manzotti S, Gesuita R, Gigante A. Matrix-induced autologous chondrocyte implantation (MACI) in the knee. Knee Surg Sports Traumatol Arthrosc 2012;20(05): 862-869

6 Gigante A, Enea D, Greco F, et al. Distal realignment and patellar autologous chondrocyte implantation: mid-term results in a selected population. Knee Surg Sports Traumatol Arthrosc 2009; 17(01):2-10

7 Gille J, Schuseil E, Wimmer J, Gellissen J, Schulz AP, Behrens P. Mid-term results of autologous matrix-induced chondrogenesis for treatment of focal cartilage defects in the knee. Knee Surg Sports Traumatol Arthrosc 2010;18(11):1456-1464

8 Enea D, Cecconi S, Calcagno S, Busilacchi A, Manzotti S, Gigante A. One-step cartilage repair in the knee: collagen-covered microfracture and autologous bone marrow concentrate. A pilot study. Knee 2015;22(01):30-35

9 Hangody L, Ráthonyi GK, Duska Z, Vásárhelyi G, Füles P, Módis L. Autologous osteochondral mosaicplasty. Surgical technique. J Bone Joint Surg Am 2004;86-A(01, Suppl 1):65-72

10 Bugbee WD, Convery FR. Osteochondral allograft transplantation. Clin Sports Med 1999;18(01):67-75

11 Seo S-S, Kim C-W, Jung D-W. Management of focal chondral lesion in the knee joint. Knee Surg Relat Res 2011;23(04):185-196

12 Gigante A, Calcagno S, Cecconi S, Ramazzotti D, Manzotti S, Enea D. Use of collagen scaffold and autologous bone marrow concentrate as a one-step cartilage repair in the knee: histological results of second-look biopsies at 1 year follow-up. Int J Immunopathol Pharmacol 2011;24(01, Suppl 2):69-72

13 Brun P, Dickinson SC, Zavan B, Cortivo R, Hollander AP, Abatangelo G. Characteristics of repair tissue in second-look and third-look biopsies from patients treated with engineered cartilage: relationship to symptomatology and time after implantation. Arthritis Res Ther 2008;10(06):R132 
14 Strauss EJ, Galos DK. The evaluation and management of cartilage lesions affecting the patellofemoral joint. Curr Rev Musculoskelet Med 2013;6(02):141-149

15 Brophy RH, Wojahn RD, Lamplot JD. Cartilage restoration techniques for the patellofemoral joint. J Am Acad Orthop Surg 2017;25 (05):321-329

16 Du D, Sugita N, Liu Z, et al. Repairing osteochondral defects of critical size using multiple costal grafts: an experimental study. Cartilage 2015;6(04):241-251

17 Ohlsén L. Cartilage formation from free perichondrial grafts: an experimental study in rabbits. Br J Plast Surg 1976;29(03): 262-267

18 Ho TT, Cochran T, Sykes KJ, Humphrey CD, Kriet JD. Costal and auricular cartilage grafts for nasal reconstruction: an anatomic analysis. Ann Otol Rhinol Laryngol 2017;126(10):706-711

19 Obert L, Loisel F, Gindraux F, Tropet Y, Lepage D. Rib cartilage grafting in upper limb surgery: an overview. SICOT J 2015;1(19):13
20 Uppal RS, Sabbagh W, Chana J, Gault DT. Donor-site morbidity after autologous costal cartilage harvest in ear reconstruction and approaches to reducing donor-site contour deformity. Plast Reconstr Surg 2008;121(06):1949-1955

21 Arøen A, Løken S, Heir S, et al. Articular cartilage lesions in 993 consecutive knee arthroscopies. Am J Sports Med 2004;32(01): 211-215

22 Yanke AB, Wuerz T, Saltzman BM, Butty D, Cole BJ. Management of patellofemoral chondral injuries. Clin Sports Med 2014;33(03): 477-500

23 Kitaoka E, Satomura K, Hayashi E, et al. Establishment and characterization of chondrocyte cell lines from the costal cartilage of SV40 large T antigen transgenic mice. JCell Biochem 2001;81 (04):571-582

24 Skoog T, Ohlsén L, Sohn SA. Perichondrial potential for cartilaginous regeneration. Scand J Plast Reconstr Surg 1972;6(02): $123-125$ 